\title{
REDUCING COMPUTATIONAL COST OF LARGE-SCALE SIMULATIONS USING OPPORTUNISTIC MODEL APPROXIMATION
}

\author{
Stig Bosmans, Siefried Mercelis, \\ Peter Hellinckx \\ University of Antwerp \\ imec - IDLab \\ Antwerp, Belgium \\ \{ stig.bosmans, siegfried.mercelis, \\ peter.hellinckx\}@uantwerpen.be
}

\author{
Joachim Denil \\ University of Antwerp \\ Flanders Make \\ Antwerp, Belgium \\ joachim.denil@uantwerpen.be
}

\begin{abstract}
We present a dynamic model approximation strategy that allows to significantly increase computational efficiency of the simulation while maintaining proper validity. This can be used to effectively overcome the scalability constraints in state-of-the-art simulation frameworks for testing and validating large-scale systems. The method that we present leverages information theory metrics to measure the possible contribution of sub-areas in the simulation to the global behavior. This allows us to opportunistically approximate lowcontributing areas and as a result decrease the computational cost of the simulation. We present a basic traffic-simulation use-case, implemented in the Acsim simulator to validate the proposed method and are able to achieve a $33 \%$ reduction of the computational cost. Furthermore, we analyze our proposed method from a more theoretical perspective.
\end{abstract}

Keywords: Agent based simulation, surrogate modeling, traffic simulation, emergent behavior.

\section{INTRODUCTION}

In this work we look to reduce the computational complexity of large scale simulations. More specifically we focus on reducing the complexity of simulating global emergent behavior in large complex systems such as Internet of Things systems or traffic systems. An IoT system is considered a large-scale complex system, where the IoT entities and actors are considered autonomous agents that interact with each other and with the environment. This autonomous and adaptive local behavior leads to a global emergent behavior. Validating, testing and tuning such global emergent behavior is key before one can deploy such system in practice. Imagine for example a smart traffic light system, where we want to optimize the global traffic flow and reduce carbon emission. In order to validate, tune or optimize these global emergent properties, such as traffic flow, we need to simulate such system at scale. In earlier work we presented an iterative tuning and validation approach for IoT applications using micro-level agent-based simulation (Bosmans, Mercelis, Denil, and Hellinckx 2018b). Leveraging this agent-based simulation approach allows for easy prototyping and accuracy but is strongly limited in its scalability capabilities due to the large amount of interacting agent entities (Bosmans, Mercelis, Hellinckx, and Denil 2018).

To overcome the scalability constraints in simulation of global emergent properties in large-systems com- 
plex systems we look at the possibility to scale the simulation by abstracting and approximating individual models or simulations areas. Transforming certain models to a less computationally intensive abstraction/approximation, will lead to a reduction of the computational cost of the simulation which in turn allows us to increase the overall scalability of the simulation. More specifically, we present an opportunistic abstraction/approximation approach, that is able to dynamically transform low-information areas to more abstract representations. We show that by using this method we are able to reduce the computational cost by $33 \%$ in the presented traffic simulation use case.

The abstraction/approximation approach we present in this paper is mainly based on the work that has been done in the area of model abstraction which has been studied extensively during the last 20 years. Model abstraction allows us to represent models at an appropriate level of abstraction, which often comes down to making a proper trade-off between computational cost, modeling effort and accuracy. In the scope of this paper we are interested in two types of abstraction: 1) State abstraction, with this technique the behavior of individual models is replaced by more abstract abstractions/approximations. Meta-modeling (in a surrogate modelling sense) is a well-studied method to automatically apply the abstraction (Caughlin and Sisti 1997). Examples of meta-modeling techniques range from complex neural network approximations to basic lookup tables. Another technique is 2) Entity aggregation, where multiple models are replaced by a single approximated agent, that abstracts the overall behavior of the collection of models. For example, Rodriguez et. al. present various statistical techniques that can be used to aggregate simulation models (Rodriguez, Bauer, Miller, and Neher 2008).

The technique that we present in this work dynamically approximates the behavior of multiple agents in a certain area by moving from a simulation area containing multiple individual car agents to a computationally more efficient Discrete Event Simulation (DES) area. It is partly related to both meta-modeling and entityaggregation, in the sense that we can approximate multiple models by a single approximation and that we use a surrogate meta-model. Note that we only reason explicitly over the approximation dimension in this paper. As abstraction is the removal of certain properties from a model, the properties of interest for the overall simulation still contain the same amount of information (there is no approximation). Abstraction is mostly done at the same time, as meta-modelling techniques generally only keep the properties of interest.

This paper is structured as follows: in section 2 we introduce the core idea of switching between simulation formalisms in order to switch between abstraction levels. In section 3 we introduce a traffic simulation use case that will help to build intuition about the problems we're trying to solve. In section 4 we introduce the idea of opportunistically approximating simulation areas based on entropy. Section 5 shows the impact of the our technique both on computational cost and on the simulation validity. Finally, we position our approach from a more theoretical perspective.

\section{DYNAMIC MODEL APPROXIMATION DRIVEN BY INFORMATION THEORY}

To improve the overall scalability of our simulation, we propose to dynamically approximate models. Our hypothesis is that when the approximation is less detailed than the original model, the overall computational cost will be lower. However, at the same time we expect the models approximated with less detail and at a higher level of abstraction to lose information. In this paper we look to opportunistically approximate models, to reduce the computational cost while maintaining the overall validity as good as possible.

To further clarify this we will first introduce some notations:

When we consider the full set of simulation models in our simulation to be $M$, the set of low abstraction models, to be $L$ and the set of high abstraction models to be $H$. Then $M=H \cup L$. With high or low abstraction models we refer to models that are expressed at a higher or lower level of abstraction. Explicitly modelled models are considered to be part of the set of low abstraction model $L$, and the set of approximated 
models to be part of the set of high abstraction models. The diagram below illustrates the hierarchical topology of our conceptual simulation framework. The Full simulation consists of both the set of high abstraction models and low abstraction models. In the remainder of this paper we look at how to facilitate a proper transition from a low abstraction model to a high abstraction model. We want to maximize the size of set $H$ while maintaining the validity of the global simulation.

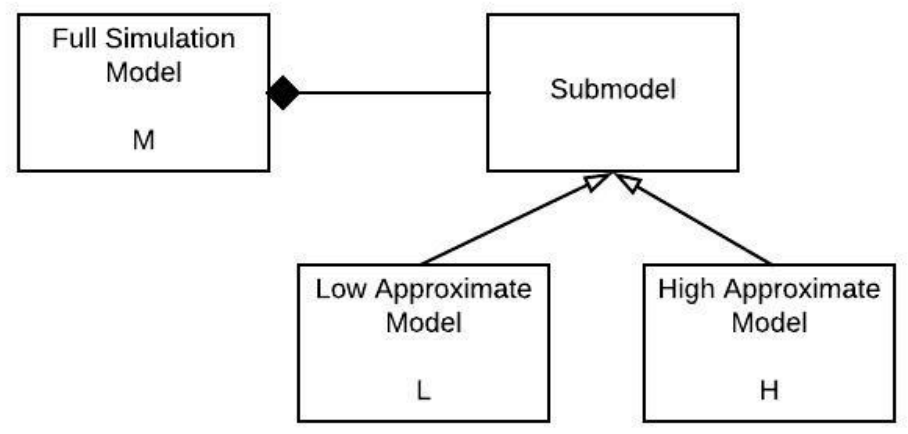

Figure 1: Topology of simulation models.

\section{A TRAFFIC USE CASE}

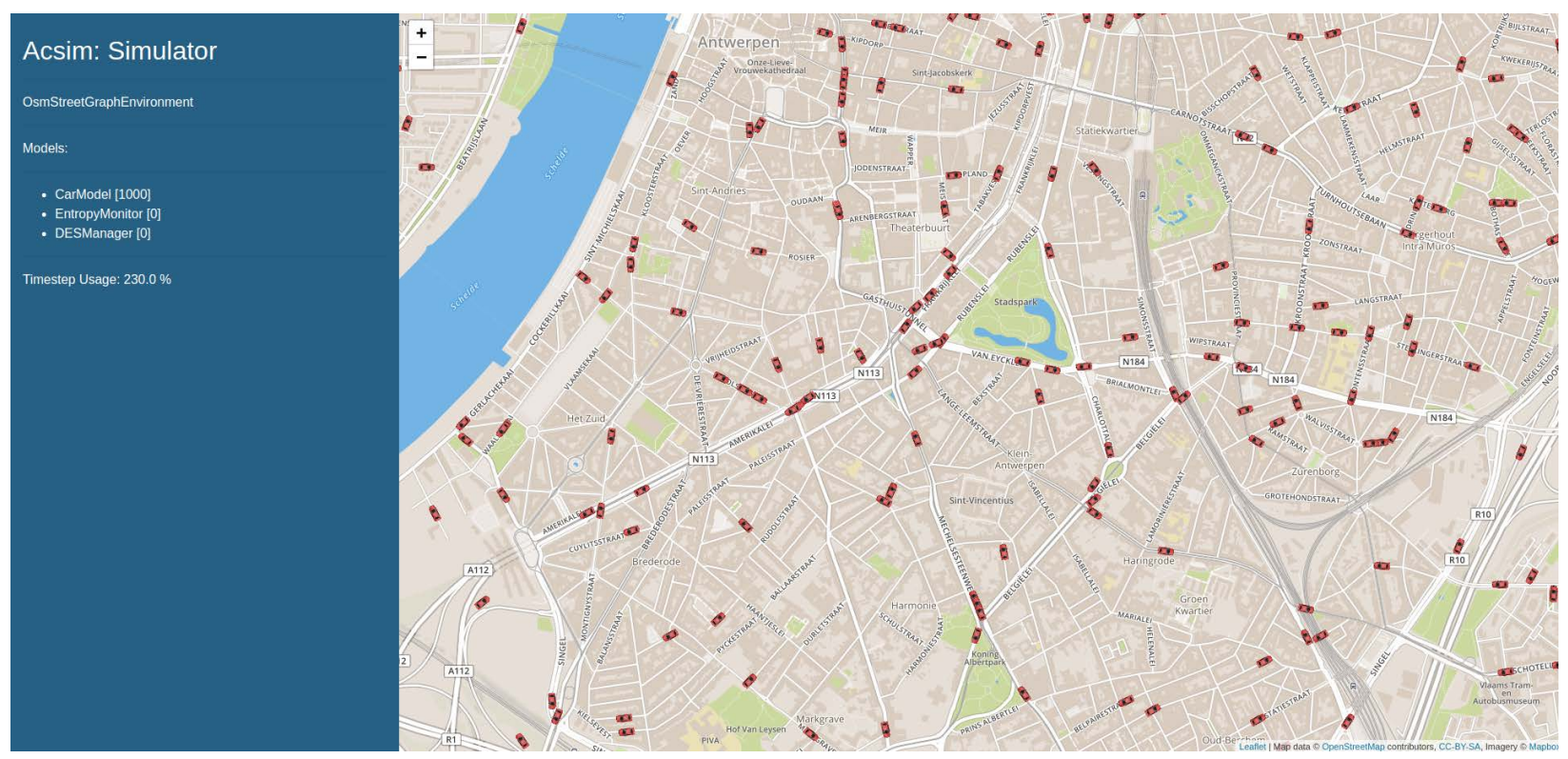

Figure 2: Traffic simulation in Acsim.

To validate the computational impact of opportunistic entropy-based transformation we introduce a basic traffic simulation use case using the agent-based IoT simulator Acsim (Bosmans, Mercelis, Denil, and Hellinckx 2018a). This will also help to build intuition of the problem we are trying to solve. The simulation use case we present, consists of 1000 cars driving at various speeds as shown in figure 2. The average speed of a car depends on the speed limit of the road the car is driving on. Based on the road type, the speed 
may deviate more or less from the given speed limit. Furthermore, we consider different speed distributions depending on the type of road the cars are driving on. These distributions are shown in figure 3 below.

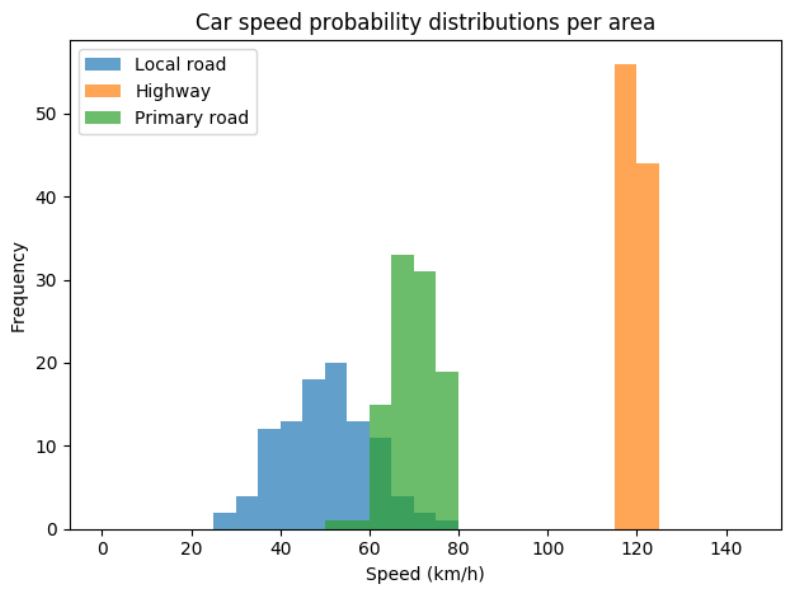

Figure 3: Car agent speed distributions per road type as observed in the simulation.

We expect more variability in the average speed on local roads due to traffic lights and road priority rules. Whereas, the variability in speed is lower on highways. These assumptions give us a proper use case to evaluate the problem we're trying to solve, where, depending on the area, different types of behavior can be observed.

To model this simulation we use the agent-based Acsim simulator. The environment is represented as a (directed) graph-based open street map environment. Roads are represented by edges and road crossing are represented by nodes. Furthermore, the environment allows to efficiently run GIS range queries and calculate routes between various locations. By default, each car is represented by a single agent. The simulation is time-driven, this means that the simulation state is updated at discrete time-steps. For example, at each time-step the car agents update their location and run some synthetic computations to simulate a realistic, constant computational load.

\subsection{Area approximation}

To move certain areas to a less-detailed approximation we allow the simulator to transform specific agentbased simulation areas to a discrete-event simulation (DES) representation of the area. The DES area approximation is also implemented as a single agent, which is running a discrete event simulation internally. This enables us to reduce the computational cost significantly but at the cost of losing local interactions. In the traffic use case a simulation area is a single road section. As can be seen in figure 4 below, each time a car agent arrives at a DES road-section area it will be deactivated. This road section agent will then schedule an internal event when the agent can leave the area. The duration depends on the agent speed, which is sampled from the historical data of agents driving on the specific area. We can say that the DES road-section area agent is approximating the behavior of incoming agents by averaging its speed. Therefore, these agents are temporarily approximated and are temporarily part of the set of high abstraction agents $H$. 

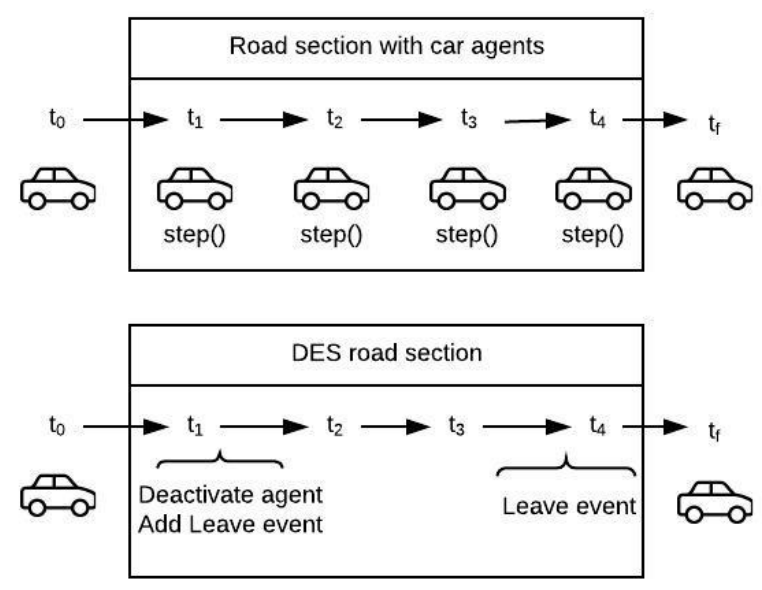

Figure 4: Road section with car agents vs. DES road section agent.

\subsection{Computational cost}

We consider the computational cost to be a measure that illustrates the computational cost to progress the simulation a single timestep $t$ to the future. Many empirical, indirect execution time measuring techniques are described in literature to measure the computational cost of a simulation. An example is the Wall Clock Time (WCT) which measures the duration of the entire simulation from start to finish. However, as priorly explained our simulation will need to run in a hybrid mode, where virtual, simulated IoT entities need to interact with real-world IoT entities or IoT middleware systems in realtime. Therefore, our goal is to satisfy the predefined amount of simulation timesteps $P$ per seconds in order that the simulation remains responsive to outside interactions. In many cases this minimum required amount of timesteps $P$ depends on the context of the simulation. Therefore, WCT is not an appropriate measure for our simulation setup. Instead, we apply the 'step-usage' metric to indirectly measure the computational cost of our simulation model. The step-usage compares the observed computation time $O_{t}$ of a single timestep $t$ of the set of all simulation models $M$ to the preferred timestep duration $d_{t}^{*}=\frac{1}{P}$.

Step - Usage $=\frac{O_{t}}{d_{t}^{*}}$.

It should be clear that this is an imperfect measure due to the fact that it may contain noise introduced by other unrelated processes but it is easy to observe and should give an accurate enough representation of the main trend we try to observe.

We can express the computational cost of the simulation at timestep $t$ as a function $C$ of set $M$, where we assume:

$C(t, M)=C(t, L)+C(t, H)$ and,

$C\left(t, H_{i}\right) \leq C\left(t, L_{i}\right)$ where $H_{i}$ is an abstract approximation of $L_{i}$ and $H_{i} \in H$ and $L_{i} \in L$. 


\subsection{Computational cost over time}

Figure 5 shows the computational cost at each time-step of the simulation. The computational cost is expressed as a step-usage ratio $C$, as introduced in section 3.2. This metric is sensitive to noise introduced by other processes, to remove this noise we applied a running average of 100 timesteps before plotting the data. It is expected that the computational cost remains constant over time. Figure 5 below shows that the computational cost during the first scenario remains constant over time as is expected. However, the computational cost exceeds the 1.0 time-step usage ratio. This could lead to the state of simulation agents being updated too late and as a consequence, to inconsistent or incorrect behavior of the simulation models.

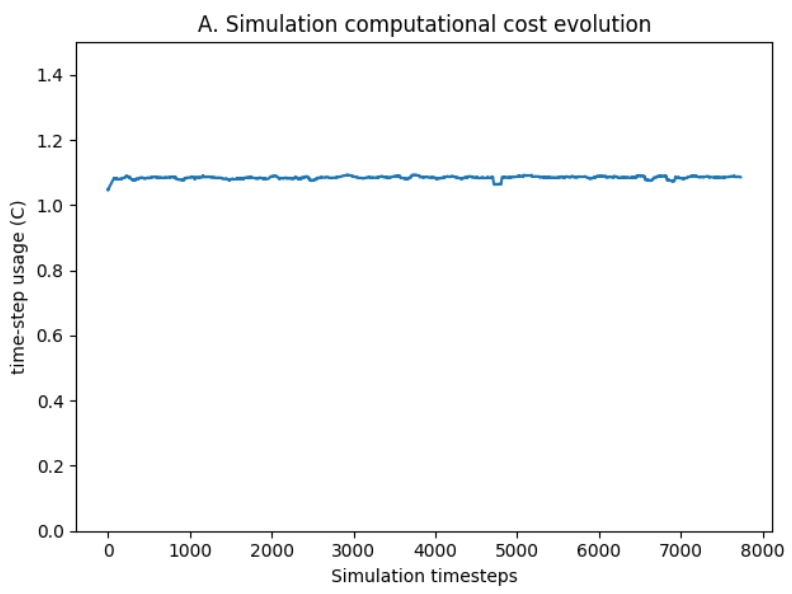

Figure 5: Computational cost over time without transformations to event-driven areas.

\section{ENTROPY BASED TRANSFORMATION}

If we look at the properties of overall global emergent behavior of a simulation, they are created by the properties of the individual simulation models. In order to maintain the validity of the overall simulation, we will have to maintain the properties of the overall simulation and therefore also the properties of individual models or collections of models. Also note that the approximations we want to make are applied on an individual property of a model. We assume that some areas of the simulation contribute more $\left(A_{H_{C}}\right)$ to the properties of the simulation compared to others. Therefore, it would be interesting to target simulation areas that contribute less to the overall behavior $\left(A_{L_{C}}\right)$. We can assume that if we transform these low-contributing areas to more abstract approximations $\left(A_{L_{C}}^{\prime}\right)$, the overall emergent behavior is less sensitive to the loss of information and the inaccuracies introduced by these approximations. In other words, we expect the simulated global behavior to be more accurate when more low contributing $A_{L_{C}}$ areas are approximated opposed to when more high contributing areas $A_{H_{C}}$.

\section{Identifying low contributing simulation areas}

When we continue to reason about this, we can conclude that after identifying the low contributing areas $A_{L_{C}}$, we can opportunistically transform these to more abstract representations which helps us to achieve our main goal, which is to reduce the computational cost while maintaining overall validity. This brings us to the core of this paper: finding a technique to identify these low contributing simulation areas. To do this, we propose to use the entropy measure from information theory (Shannon 1951). Entropy is a measure that can be used to determine the amount information or amount of randomness in a dataset. It is expressed by 
formula 1 below:

$$
E=\sum_{i=1}^{n}\left[p_{i} \log _{2}\left(p_{i}\right)\right]
$$

There is a connection between the amount of information that can be observed in a given simulation area and its contribution to the global emergent behavior of the entire simulation. Furthermore, low information areas are often easier to predict and therefore, the properties of a more abstract approximation of the area are easier to be maintained after a transformation. Another advantage of using entropy is that it is easy to measure. We can easily extend the simulation to keep track of the entropy value of individual areas. When comparing this with the entropy of the global simulation is should allow us to dynamically transform low-entropy areas to higher abstraction levels. This will decrease the computational cost while maintaining the validity. Furthermore, entropy is a general-purpose metric, it can be used for multiple simulations in multiple contexts without requiring expert domain knowledge. In related work, P. Lamarche, leverages similar information theory inspired techniques to evaluate the information loss of abstractions in the context of a multi-agent system (Lamarche-Perrin, Demazeau, and Vincent 2013). The entropy-based technique we present in this work, is similar but is not used to evaluate the information loss after the abstraction, but rather estimates the possible impact on the global simulation behavior when a given area is abstracted or transformed.

\section{Measuring entropy in the traffic use case}

To decide when and where we can transform standard areas to DES areas we need to extend the simulation framework used in section 3 to measure entropy levels. We do this by regularly measuring the speed driven at each area and categorize these measurements at intervals of $5 \mathrm{~km} / \mathrm{h}$. This allows us to measure discrete probabilities and as a consequence to measure entropy as expressed by equation 1 . When the entropy level exceeds a certain threshold we transform the standard area to a DES area. We use a heuristic to determine the threshold, it is set $1 / 3^{\text {th }}$ of the global entropy value. So an area will switch from a standard to DES simulation when the area's entropy is below $1 / 3^{\text {th }}$ of the global entropy value. In the section below we present the results on the computational cost of the simulation after applying entropy-based transformations.

\section{RESULTS}

In this section we verify the impact of our entropy-based approximation strategy on both the computational cost and the validity of the traffic simulation use case. The setup is similar to the results presented in section 3.3, except this scenario does perform transformations to event-driven areas based on the entropy measures introduced in the previous section.

\section{Transforming time-based simulation areas to event-driven areas}



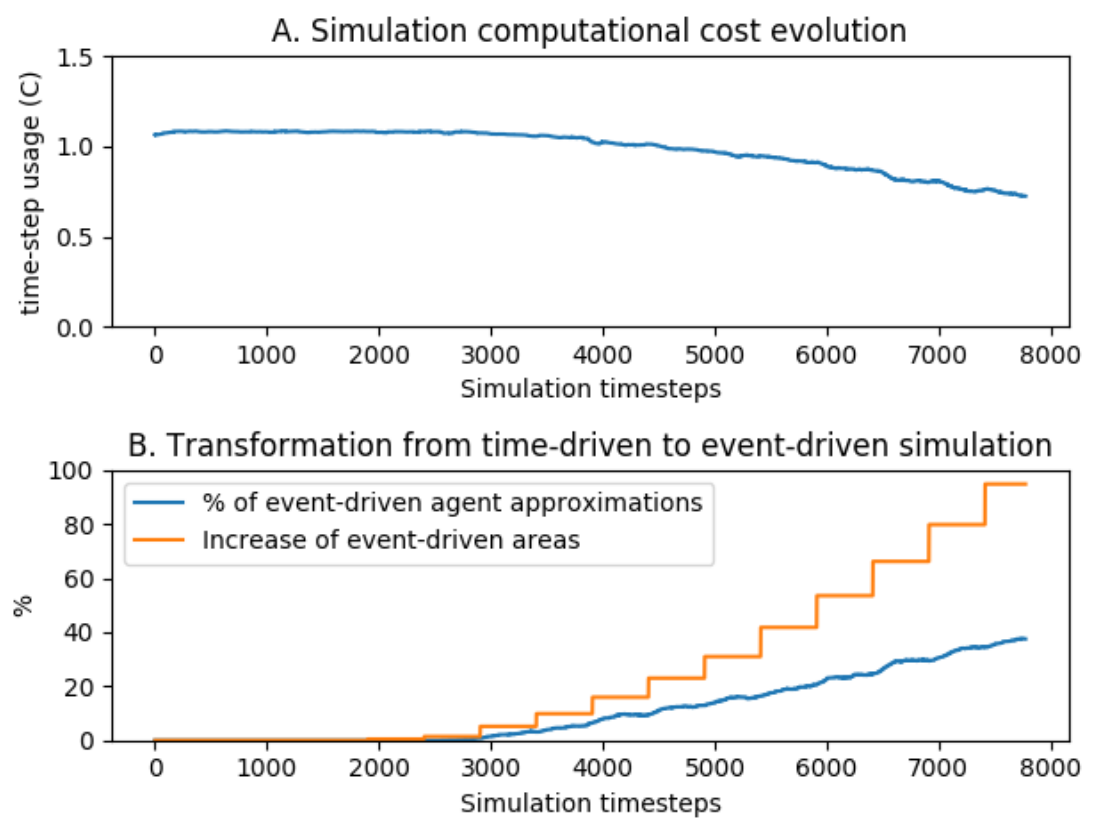

Figure 6: Time- to event-driven transformations.

Figure 6.A shows the computational cost over time of the second scenario. Figure 6.B shows the evolution of standard areas being transformed to DES areas over time. As can be seen at the beginning of the simulation all areas are running in the standard formalism. However, after about 2000 timesteps the simulation has gathered enough entropy measurements and starts to slowly transform areas to DES areas. Note that this only occurs when enough measurements are gathered and when the local area entropy is below $1 / 3^{\text {th }}$ of the global entropy value. This directly leads to more agents being approximated by the event-driven formalism. As a consequence, the computational cost decreases. After 8000 simulation time-steps, 202 low-entropy, areas have been approximated by DES areas and 37\% of agents are approximated by these areas. Furthermore, the time-step usage ratio $C$ decreases significantly from 1.08 at the beginning of the simulation to .72 after about 8000 timesteps. That is a $33 \%$ decrease in computational cost, which can be used to scale up to even more agents. Furthermore, after about 4500 timesteps the computational cost of the simulation is below 1.0 time-step usage ratio, which is considered to be safe.

\section{Impact of approximation strategies on simulation validity}

To test the impact of approximating simulation areas on the overall validity of the simulation we compare a random area selection strategy versus an entropy-driven area selection strategy as presented in section 4 . To quantify the validity we will monitor a global simulation property that we expect to be invariant after multiple runs of different simulations. The invariant property selected for the traffic use case is the average distance driven per car. When no approximations are applied we expect the difference in this average distance to be minimal. However, after approximating certain simulation areas we expect this difference to be larger because we're losing accuracy in individual car speed. The graph in figure 7 shows the average error in distance at each simulation timestep for all active agents. The random transformation strategy is our baseline, it approximates random simulation areas with a discrete event area simulation. The reason we selected this as the baseline is that there are no other techniques described in literature that can be used in the context of this problem. The entropy based transformation strategy only approximates simulation areas with a low entropy value as described in section 4. After about 2000 timesteps the first areas are starting to be approximated. After 8000 timesteps the error in distance of the random strategy is significantly larger 
compared to the entropy based strategy. Therefore, we can conclude that the validity of the simulation is substantially larger when we use the entropy-driven strategy compared to a random uninformed strategy. Note that for each traffic simulation the routes driven by the agents are deterministic, but the speed of the agents are not. The speed is sampled from a random normal distribution with a mean that corresponds to the speed limit of the road the car is driving on, as shown in figure 3. Therefore, the distance driven by each car will be slightly different for each run of the simulation. In order to only visualize the average error introduced by the random approximation strategy and the entropy approximation strategy, we subtracted the average error of simulation runs that didn't approximate any areas. This explains why the strategy and entropy graphs in figure 7 are not exactly zero before 2000 timesteps, as would expected in a fully deterministic simulation run.

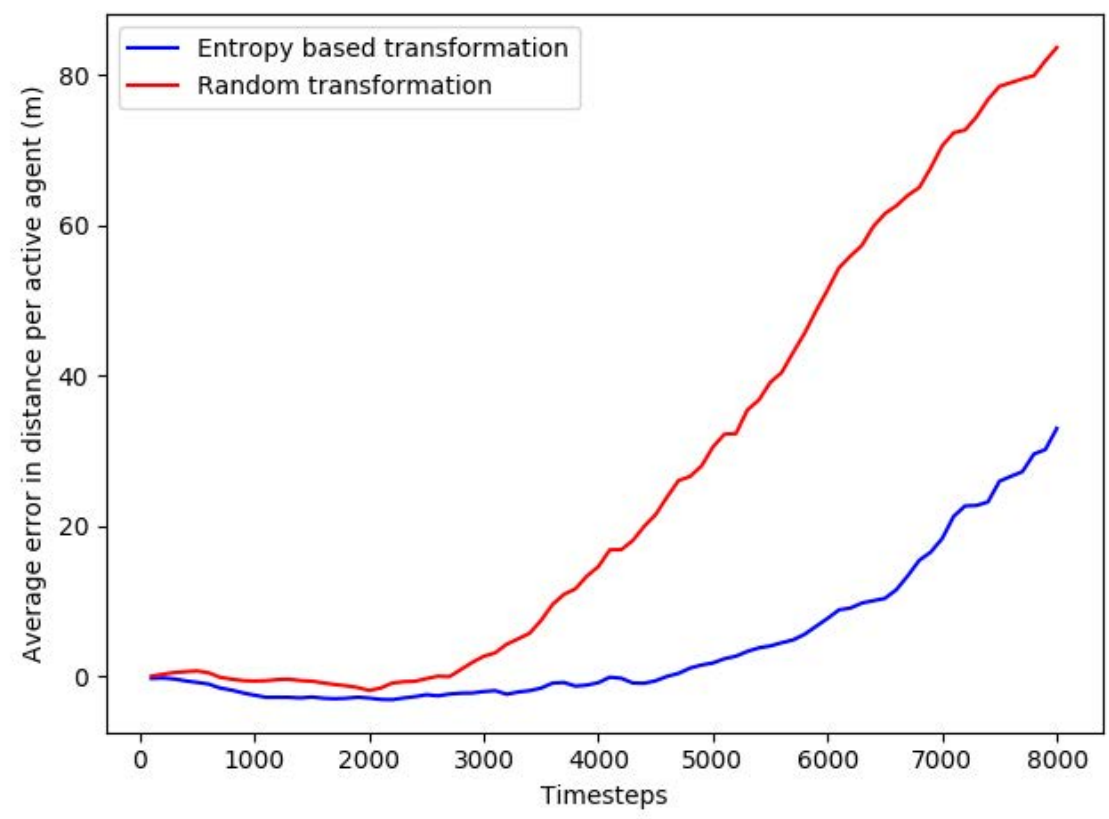

Figure 7: Approximation error after a random and entropy-driven transformation strategy - average of multiple runs of various simulations.

\section{DISCUSSION}

In this section we present the proposed method in a more rigorous way. From a more theoretical point of view we can consider the assumptions, guarantees and constraints that are inherent in individual simulation models or agents to be part of a frame. An experimental frame is defined by Zeigler et. al. as a specification of the conditions under which the system is observed or experimented with (Zeigler, Kim, and Praehofer 2000). A frame provides the necessary contextual information for a model. A frame specifies where the model is valid and the results are considered valid. More recently Traore and Muzy (Traoré and Muzy 2006) and Denil et al. (Denil, Klikovits, Mosterman, Vallecillo, and Vangheluwe 2017) worked further on the idea of model frames.

In our work, our simulation model is a composition of different sub-models. The sub-models all have specific frames to denote the validity. The composition also has a frame to specify for which properties this is a valid model. When a sub-model is replaced by another model with the same frame or a bigger frame (a frame with a model that is valid in a broader context, and gives more accurate results), the composition 
is still valid. This is because the properties in the sub-model on which the composition relies are at the same approximation (or better). However, this is not what we consider in our work. We expect that the behavior after the transformation to a more approximated model $\left(M_{L A}=>M_{H A}\right)$, to be similar (within given boundaries) and is included under the same frame of the overall composed model.

In figure 8 the difference in model behavior after transformation is represented by a distance value $D$, which is the difference between two performance values (that result in the property) $\mathrm{P}$ and $\mathrm{P}$ '. Ideally, this distance is minimal.

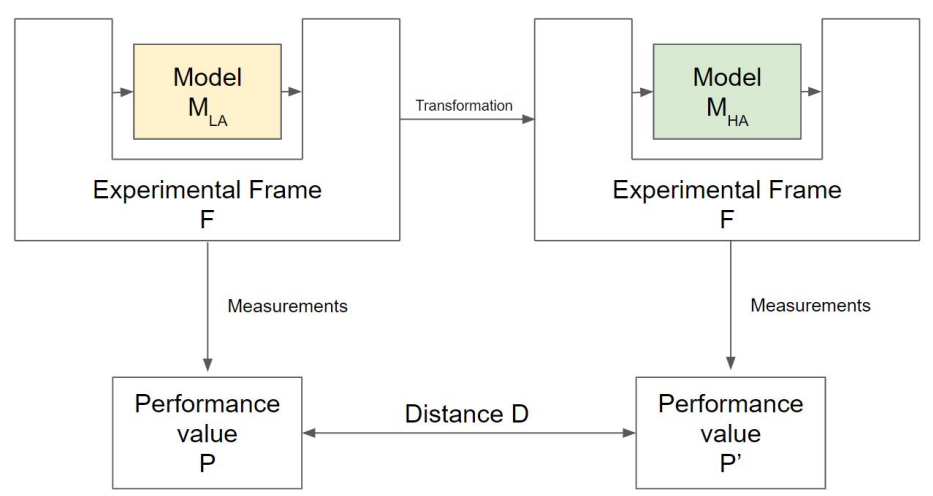

Figure 8: Model transformations and experimental frames.

We can more explicitly reason about this by looking into the different properties of the sub-model and composed model. We use the framework proposed by Barroca et al (Barroca, Kühne, and Vangheluwe 2014) to look at properties of models. A model defines its meaning through a semantic mapping, notated as [[.]]. With simulation this usually results in a set of traces. These traces are related to certain performance values (e.g. the speed of a specific car on a certain road section). These performance values are translated to the ontological domain where they are transformed to a Boolean value (e.g. no traffic congestion exist in the city). The ontological domain are the properties we are interested in.

When reasoning about the sub-models of our simulation, we need to be aware of the properties of interest of the sub-models on the properties of the composed model. Figure 9 shows this: both the sub-model $M_{L A}$ and its approximation $M_{H A}$ have a semantic mapping to its corresponding performance values as illustrated in figure 8. Both of these performance values ( $\mathrm{P}$ and $\mathrm{P}$ ') need to hold in the ontological domain in order for the composed model and its simulation to be considered valid.

The previous allows us (in theory) to explicitly reason about the distance function required for the two models to still be valid. This can be used to our advantage as in mostly more approximate models are usually better with respect to computational cost. We thus need to find a trade-off where the computational cost is much better but at the same time the distance value $\mathrm{D}$ is within the bound of the validity so that the global simulation remains within acceptable boundaries.

The approach that we present in our work replaces the use of this explicit reasoning over distance and ontological properties with an entropy measure over the performance values needed by the overall simulation. It is a pragmatic metric and we assume that the representation of amount of information is indirectly related to the validity of the model. A model that does not produce a lot of information with respect to a certain performance value, will also not have a big difference in the distance function between a more or less approximate model. 


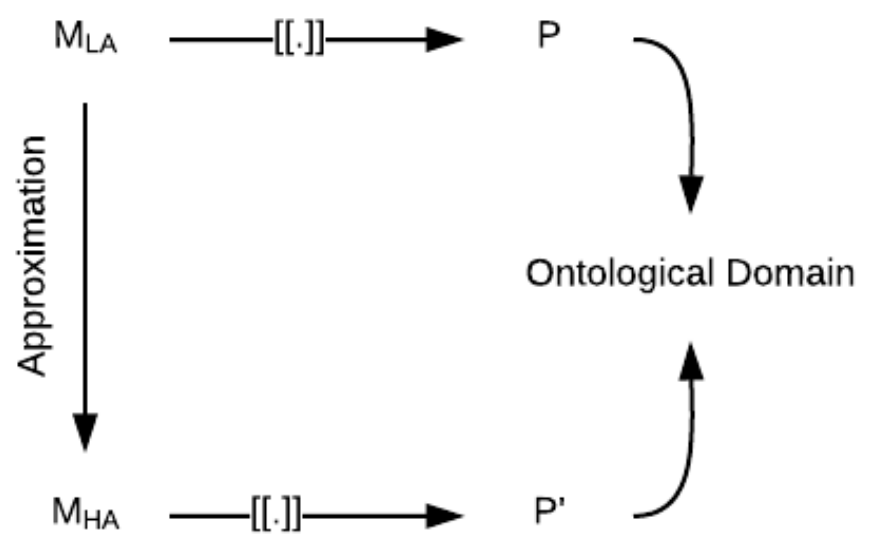

Figure 9: Semantic transformations to be evaluated in ontological domain.

Entropy is however limited as a metric because it only represents a statistical measure which loses domainrelated context about the original model. Also, our experiments were limited to unimodal distributions, which is unrealistic in practice. Many models can only be represented by a multimodal distribution. For example, the unimodel highway velocity distribution presented in our use case will in many cases only be valid outside rush-hour when there are no traffic jams. When we want to incorporate rush-hour in the highway distribution the outcome will become a multimodal distribution. The measured entropy of this distribution will be much higher, and as a result of this our proposed method will not be effective. Either, the entropy will be too high to move to another model, or the model will not produce correct results with respect to its properties (e.g. on the highway it will let the cars drive around $60 \mathrm{~km} / \mathrm{h}$ instead of $20 \mathrm{~km} / \mathrm{h}$ during rush hour or $120 \mathrm{~km} / \mathrm{h}$ outside rush hours). Instead we could measure entropy-levels based on contextinformation. For example, when we make a distinction between the highway velocity distributions based on time our entropy value will become valid again. This will then result in a unimodal distribution at rush hour and a unimodel distribution after rush hour, opposed to a multimodal distribution when time is not taken into account.

\section{CONCLUSION}

In this work we presented a method to opportunistically increase abstraction levels of low-information areas to decrease the computational cost of our simulation. Our hypothesis is that we can use entropy as a metric to identify these low-information areas and that the global simulation behavior is less sensitive to noise in these low-information areas. This makes these areas ideal to approximate by a less computationally intensive proxy while maintaining the overall simulation behavior. Our experiment shows that we're able to reduce the computational cost by about $33 \%$ and that we're able to reduce the global approximation error when we opportunistically transform simulation areas with a low entropy value. This highlights the potential of our approach and it shows that our technique succeeds in reducing the computational cost while maintaining the validity of the simulation to a certain level. In future work we want to evaluate our method in various different, more complex scenario's. This will help to better identify the limitations of the proposed methodology and work towards a more generic strategy. We will also analyze the impact of the 
threshold heuristic to the resulting validity or computational cost. Furthermore, we will evaluate additional approximation techniques.

\section{REFERENCES}

Barroca, B., T. Kühne, and H. Vangheluwe. 2014. "Integrating Language and Ontology Engineering.". In MPM@MoDELS, pp.77-86. Citeseer.

Bosmans, S., S. Mercelis, J. Denil, and P. Hellinckx. 2018a. "Challenges of modeling and simulating Internet of Things systems". In International Conference on P2P, Parallel, Grid, Cloud and Internet Computing, pp. 457-466. Springer.

Bosmans, S., S. Mercelis, J. Denil, and P. Hellinckx. 2018b. "Testing IoT systems using a hybrid simulation based testing approach". Computing, pp. 1-16.

Bosmans, S., S. Mercelis, P. Hellinckx, and J. Denil. 2018. "Towards evaluating emergent behavior of the internet of things using large scale simulation techniques (wip)". In Proceedings of the Theory of Modeling and Simulation Symposium, pp. 4. Society for Computer Simulation International.

Caughlin, D., and A. F. Sisti. 1997. "Summary of model abstraction techniques". In Enabling Technology for Simulation Science, Volume 3083, pp. 2-14. International Society for Optics and Photonics.

Denil, J., S. Klikovits, P. J. Mosterman, A. Vallecillo, and H. Vangheluwe. 2017. "The experiment model and validity frame in M\&S". In Proceedings of the Symposium on Theory of Modeling \& Simulation, pp. 10. Society for Computer Simulation International.

Lamarche-Perrin, R., Y. Demazeau, and J.-M. Vincent. 2013. "How to build the best macroscopic description of your multi-agent system?". In International Conference on Practical Applications of Agents and Multi-Agent Systems, pp. 157-169. Springer.

Rodriguez, J. D., K. W. Bauer, J. O. Miller, and R. E. Neher. 2008. "Building prediction models of large hierarchical simulation models with artificial neural networks and other statistical techniques". In Visual Information Processing XVII, Volume 6978, pp. 69780M. Int. Society for Optics and Photonics.

Shannon, C. E. 1951. "Prediction and entropy of printed English". Bell system tech journal vol. 30.

Traoré, M. K., and A. Muzy. 2006. "Capturing the dual relationship between simulation models and their context". Simulation Modelling Practice and Theory vol. 14 (2), pp. 126-142.

Zeigler, B. P., T. G. Kim, and H. Praehofer. 2000. Theory of modeling and simulation. Academic press.

\section{AUTHOR BIOGRAPHIES}

STIG BOSMANS is a PhD student at the faculty of applied engineering at the University of Antwerp (UA). His email address is stig.bosmans@uantwerpen.be.

SIEGFRIED MERCELIS is a postdoc researcher at the faculty of applied engineering at UA. His email address is siegfried.mercelis@uantwerpen.be.

PETER HELLINCKX is an assistant professor at the faculty of applied engineering and head of the department Electronics-IT at UA. His email address is peter.hellinckx@ uantwerpen.be.

JOACHIM DENIL is an assistant professor at the faculty of applied engineering at UA. His email address is joachim.denil@uantwerpen.be. 